\title{
Joint Power Control and Scheduling Algorithm for Wi-Fi Ad-hoc Networks
}

\author{
Sangho Oh*, Marco Gruteser*, Daniel Jiang ${ }^{\dagger}$, Qi Chen ${ }^{\dagger}$ \\ *WINLAB, Rutgers University, NJ 08902-3390, USA \\ Email: \{sangho, gruteser\}@winlab.rutgers.edu \\ ${ }^{\dagger}$ Mercedes-Benz Research \& Development North America, Inc, 850 Hansen Way, Palo Alto, CA, USA \\ Email: \{daniel.jiang, qi.chen\}@daimler.com
}

\begin{abstract}
Power control is a widely used technique in improving the throughput and the quality of services in wireless communications. In this paper, a power control method based on a utility maximization method is applied to Ad-hoc networks built on Wi-Fi radios. First, a fully distributed power control algorithm is developed considering the different characteristics of discrete multi-rate $\mathrm{Wi}-\mathrm{Fi}$ radios, then a novel joint power control and scheduling algorithm is proposed to maximize the network throughput without sacrificing fairness. The proposed algorithm has a linear complexity degree while other utility based sub-optimal algorithms have exponential complexity. Lastly, as a preliminary step for an application for real Wi-Fi networks, the SINR model of Wi-Fi radios is verified through an experimental research based on the power capture effects.
\end{abstract}

\section{INTRODUCTION}

Considering the rapid growth of Wi-Fi networks and the densely installed Wi-Fi hotspots in urban areas, power control techniques should be actively applied to explore limited radio resources. Besides the gains of network throughput, for energy constrained wireless end user devices power control can also extend the battery usage time.

In wireless communication systems, power control has been throughly studied over decades aiming to enhance the network efficiency and the quality of service. Through the proper assignment of transmitting powers of wireless devices, it is possible to increase network throughput by reducing the level of mutual interference among devices. From the perspective of network throughput optimizations, power control is identical to the resource allocation by solving network-wide utility maximization (NUM) problems [1], [2], [3], [4], [5].

Although utility based power control methods produce optimal network throughputs, they require frequent message exchange between nodes and also easily produce severe unfairness to individual node's throughput [6] depending on the topological conditions of the network. When mutual interference between nodes is too high and the rate region of the network is convex, a number of nodes are severely penalized. The fairness problem can be solved by joint scheduling and power control methods that guarantee minimum resource allocation by scheduling the nodes penalized. By orthogonalizing the communication links - a transmitter and a receiver node pairs - that produce high interference to other nodes, the rate region becomes convex achieving higher network throughput.
However, the joint scheduling and power control problem is known to be a NP-complete problem [7] that requires a global knowledge on the network, which makes it difficult be applied to Ad-Hoc networks that do not have a central coordinating node. Another issue in applying power control to Wi-Fi systems is the possible discrepancy between theatrical SINR thresholds and realistic values. For some low power wireless devices, it is known that the standard SINR model cannot be applied [8]. Therefore, the verification of SINR models for commodity 802.11 radios is a prerequisite for the practical application of power control algorithms. Through the extensive experiments on the power capture effects exploiting synchronous packet collision ${ }^{1}$, we measure the exact reception thresholds per data rate, and verify the SINR model of actual Wi-Fi network cards. The measured SINR thresholds are also used for the calibration of simulations.

In this paper, we review the issues that arise in applying the utility-based power control methods to Wi-Fi radios, which are related to the convexity of the utility function and the feasibility of rate regions. To tackle the complexity issues related to joint scheduling and power control, we propose Joint Power control and Scheduling Algorithm (JPSA). The proposed algorithm employs a simple greedy algorithm that autonomously groups nodes into a number of subgroups for scheduling for a time division multiple access. The algorithm is based on the Lagrangian method that is customized for staircase utility function of multi-rate Wi-Fi radios. Although its performance is not optimal, it has the following advantages over other NUM based joint power control and scheduling algorithms which have exponential complexity over the size of rate set and number of nodes.

- Linear complexity: Complexity $C(N)$ linearly increases with the number of nodes $(\mathrm{N})$ and is independent of the size of rate set $(\mathrm{R})$

- Distributed control: The algorithm is fully distributed and requires very limited message exchange between nodes.

This paper is organized as follows. In Sect.II, we review the related research on NUM based power control methods. We introduce system models and identify the problems. Then, Sect.IV follows with the detailed explanation of our proposed

\footnotetext{
${ }^{1}$ Power capture refers to the correct decoding of a frame even though a multiple access collision occurred.
} 
JPSA. The simulation setup and the results are presented in Sect.V and Sect.VI. Then we show the experimental results using Wi-Fi radios in Sect.VII. The conclusions are made in Sect.VIII.

\section{RELATED WORK}

Utility based power control methods have been widely studied in cellular systems that are characterized by centralized base stations and mobile nodes connected to them. One of the key power control algorithm was developed in [9]. Authors show that the utility-maximization problems for an optimal cell throughput can be solved in a distributed mechanism by introducing a price function, which is reduced to a distributed power control algorithm for individual nodes. For multi-cell environments, Ji et al. solved power control problems based on a convex utility function [3], and a distributed power control algorithm based on load-spillage is provided in [4]. Han et al. solved power control problem using a non-linear price function [10] for uplink CDMA system. These papers assume centralized systems that a base station broadcast system information to mobile stations, and they do not discuss the fairness issue between users. In [1], Xiao et al. proposed an algorithm that adaptively modifies the price function to increase the fairness of users using a non-convex Sigmoid like utility function. Allowing soft SINR thresholds authors alleviate the fairness problem, however, this scheme requires a tuning process of the parameters.

In Ad-hoc networks, power control has mainly been applied for interference mitigation on a per-link basis to increase both energy efficiency of nodes and the network capacity [11], [12], [13], [14], [15]. In [16], [17], [18], adaptive rate control is jointly considered with power control methods. In terms of per-network basis power control methods that primarily maximize network throughput, Vivek et.al. propose a crosslayered approach by jointly optimizing carrier sensing range and the power levels of nodes [19], and Narayanaswamy combines a power control algorithm with a routing protocol for network wide coordinations [20]. Coping with the fairness issues raised in network throughput maximization [6], joint power control and scheduling approach is made by solving network-wide utility maximization (NUM) problems in [21], [7]. However, these algorithms not only require a global knowledge on the network, but also the complexity of the algorithms increases exponentially with the size of discrete rate set and the number of nodes.

\section{System Model ANd Problem}

In Ad-hoc peer-to-peer networks, as shown in Fig 1, transmitter nodes and receiver nodes make communication link pairs. In our unicast communication model, each communication link, $l_{i}$, is assumed to have one transmitter node $s_{i}$ and one receiver node $r_{i}$, which are not shared by any other link, and $G_{i i}$ is wireless channel gain for the connection.

In interference limited communication systems such as CDMA and OFMDA systems, more than one communication pair are allowed to transmit data simultaneously. In such

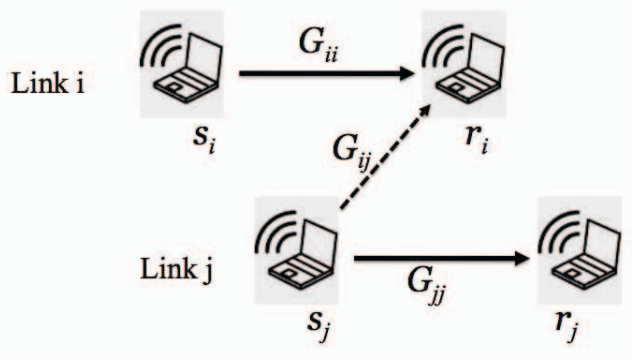

Fig. 1. Ad-hoc peer-to-peer communication model (Unicast mode)

cases, a link capacity is limited by the interference caused by the transitions from its neighboring links. The Signal to Interference and Noise Ratio (SINR), $\gamma_{i}$, at node $r_{i}$ can be represented by the standard interference model in (1), which is a function of $M$ interfering nodes' transmission power vector $\boldsymbol{p}=\left[p_{1}, \cdots, p_{i}, \cdots, p_{M}\right]^{t}$, where the transmission power is limited by a maximum value of $\bar{p}$. The amount of interference to $r_{i}$ is related to the network channel gain matrix denoted by $G_{i j}$ from the transmitter node $s_{j}$ from link $j$ to $r_{i} . N_{i}$ is the thermal Gaussian noise component at $r_{i}$.

$$
\gamma_{i}(\boldsymbol{p})=\frac{G_{i i} p_{i}}{\sum_{j \neq i} G_{i j} p_{j}+N_{i}}
$$

We can rewrite (1) as follows with the normalized channel gain matrix $F_{i j}$ and the normalized thermal Gaussian noise $\eta_{i}$ for $F_{i j}=\frac{G_{i j}}{G_{i i}}, \eta_{i}=\frac{n_{i}}{G_{i i}} . R_{i}$ is the total amount of interference to node $r_{i}$.

$$
\gamma_{i}(\boldsymbol{p})=\frac{p_{i}}{\sum_{j \neq i} F_{i j} p_{i}+\eta_{i}}=\frac{p_{i}}{R_{i}}
$$

Applying NUM techniques to wireless communications, there are number of ways of defining utility functions depending on the applications of the service. Voice quality, data rates, network efficiency, and network throughput are examples of utility functions. In Wi-Fi Ad-hoc networks, we are defining the utility function as the sum of data rates of individual links, then the utility maximization problem can be described as following equations.

$$
\begin{array}{ll}
\operatorname{maximize} & \sum_{i} U_{i}\left(\gamma_{i}(\boldsymbol{p})\right) \\
\text { subject to } & 0 \leq \boldsymbol{p} \leq \overline{\boldsymbol{p}}
\end{array}
$$

Here, the optimization variable is power of each node, $p_{i}$. By controlling $p_{i}$, we can maximize the network throughput. However, not all power assignments are feasible due to the mutual interference in the networks. Because the change of power in one the link affects all other links and their data rates, nodes are required to coordinate to maximize the network throughput. 


\section{A. Applying NUM based power control to Wi-Fi radios}

Applying NUM methods for Ad-hoc networks for maximizing network throughput using minimum transmission power, the utility $U_{i}$ is defined as the data rate of Wi-Fi radios. To allow the variable rate of $\mathrm{Wi}-\mathrm{Fi}$ systems that changes depending on SINR values, we set the utility function as a staircase function of $\gamma$ as shown in Fig. 2 according to the 802.11a specifications. However, it is hard to directly apply NUM methods to the multi-rate Wi-Fi radios as NUM is based on convexity of the utility function while stair-case multi-rate function is a non-convex function which is discontinuous at each of rate transitions. Approaches made in [21], [7] for this discrete utility function increase the algorithm complexity exponentially with the size of the rate set.

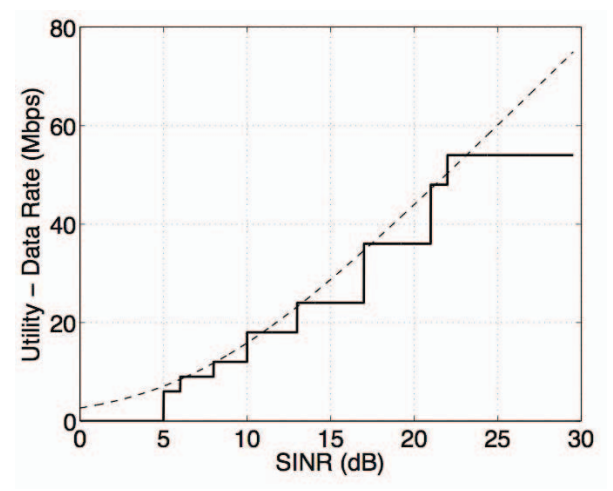

Fig. 2. Staircase Utility function

Another issue in applying power control methods to real Wi-Fi radios comes from the fact that Wi-Fi radios are not designed to allow simultaneous transmissions. Wi-Fi radios are based on the carrier sensing medium access control mechanism, and are only rely on the carrier sensing threshold to determine the clearance of the channel. If the standard SINR model does not apply for Wi-Fi radios, it is not possible to exploit power control methods in Wi-Fi systems. Therefore, the SINR model of Wi-Fi radios should be verified before a power control scheme is applied.

\section{B. Joint power control and scheduling}

Solving a standard NUM problem in assigning powers to each transmitters, the utility maximization problem (4) can be rewritten as the following dual decomposition problem [10], which can be solved in each node in a distributed way.

$$
D(\lambda)=\max _{\boldsymbol{p}} \sum_{i}\left(U_{i}\left(\gamma_{i}(\boldsymbol{p})\right)-\lambda_{i} p_{i}\right)+\sum_{i} \lambda_{i} \bar{p}_{i}
$$

Using gradient update method, price, $\lambda$ is updated by the following equation.

$$
\lambda_{i}^{(n+1)}=\left[\lambda_{i}^{(n)}+\alpha\left(p_{i}^{(n)}-\bar{p}_{i}\right)\right]^{+}
$$

In the system of a convex utility function, powers only converge to either zero or to the maximum power, which is called as bang-bang type power control that either turn off nodes or make nodes transmit in full power. The is due to the fact that the price, $\lambda$, converges only when power approaches to the maximum value, $\bar{p}_{i}$, and utility function monotonically increase with $p_{i}$ unless the node is turned off $\left(p_{i}=0\right)$.

This causes serious fairness issues as nodes under large interference are forced to be turned off as they are hard to increase their utility for the same price of $\lambda$. There are a number of approaches to alleviate this unfairness problem. One method is to adjust the price function and adaptively adjust the price values as in [1]. However, this method requires calibration of the parameters, which is not a fundamental solution for the fairness issue.

Simple NUM based power control algorithms maximize network throughput, however, nodes will experience severe unfairness as the links produce large spillage are turned off by the algorithm to maximize the network throughput [4]. Considering the fairness issue, when the size of the network is large and their inter-distances are close enough to generate large interference to each other, it is required to schedule the transmissions of nodes for a multiple access to maximize the network throughput. Link scheduling is to orthogonalize links in time domain or in frequency domain to increase both the fairness and the aggregate throughput of the network simultaneously.

We are considering a scheduling method that orthogonalize links in time domain without a need for extra frequency. By separating the links into more than one groups, it is possible to increase the total aggregate throughput by alleviating mutual interference. However, scheduling the transmission while simultaneously power controlling each link is a NP-complete problem, which is very hard to solve even in a centralized system.

For the link set $L=\left\{l_{1}, l_{2}, \cdots, l_{M}\right\}$, we can divide the links into more than two subgroups of links, $C_{1} \cup C_{2} \cup$ $\cdots \cup C_{k}=L$ for the channel access in different time slots. Thus the scheduling problem is reduced to assigning links to each subgroup while applying power control algorithm to each subgroup maximizing the aggregate network throughput.

\section{Joint Power Control And Scheduling Algorithm}

We propose a Joint Power control and Scheduling Algorithm (JPSA) based on Utility maximization, which can provide a significantly improved throughput without sacrificing the fairness of users.

In Wi-Fi systems, applying convex optimization techniques for the distributed power control, the first problem is the complexity of the algorithm caused by the discrete utility function of multi-rate. The complexity increases exponentially with the size of rate set. In addition to this, we cannot apply a gradient method to solve the maximization problem because the utility function is non differentiable due to its discontinuity at its rate transition SINR thresholds. Although we can apply a sigmoidal function instead of staircase function in approximating the utility functions [1], the sigmoidal function is still 
a non-convex function, which is hard to be solved by standard methods used for general convex optimizations problems.

\section{A. Power control with a staircase utility function}

Because there is no central coordinator in Ad-hoc networks, the scheduling and power control should be made with minimum exchange of information among nodes in the networks. We are assuming zero message exchange for power control. However, for scheduling, we need to allow a minimal information sharing between nodes to control the slot numbers and the timing of slots to avoid collisions between nodes.

Although the utility function is non-convex and is not differentiable, we can still exploit the concept of social welfare based on the price of the resource usage in the standard utility maximization problems. In (III-B), the distributed algorithm uses price, $\lambda$, in coordinating nodes to socially maximize the network throughput. The price prevents each node from selfishly exhausting air resources by increasing their transmission power to the maximum value.

Applying the staircase utility function of $\mathrm{Wi}-\mathrm{Fi}$ radios, nodes do not need to increase their transmission power to the maximum as their utility does not linearly increase with their transmission power. Once the transmission power reaches a certain threshold for a data rate, its utility does not increase until the power reaches to the next threshold. This is problematic as $\lambda$ always diverge to infinity, and the power never converges to any value. Thus, we modified the update algorithm of $\lambda$ to the following equation to suppress its divergence problem. $\lambda$ decreases as the number of iteration increase as it is divided by the iteration step $k$.

$$
\lambda_{i}^{(n+1)}=\left[\lambda_{i}^{(n)}+\frac{\alpha}{k}\left(p_{i}^{(n)}-\bar{p}_{i}\right)\right]^{+}
$$

In each node, with the updated price $\lambda_{i}$, the utility is calculated and the power is adjusted to increases its own utility value $U_{i}$, which is affected by the interference from other transmitter's transmissions power $\boldsymbol{p}_{\boldsymbol{i}}$.

$$
p_{i}^{(n+1)}=\left[\arg \max _{p_{i}} U_{i}\left(\gamma_{i}\left(p_{i}, \boldsymbol{p}_{\boldsymbol{i}-}^{(\boldsymbol{n})}\right)\right)-\lambda_{i}^{(n)} p_{i}\right]^{+}
$$

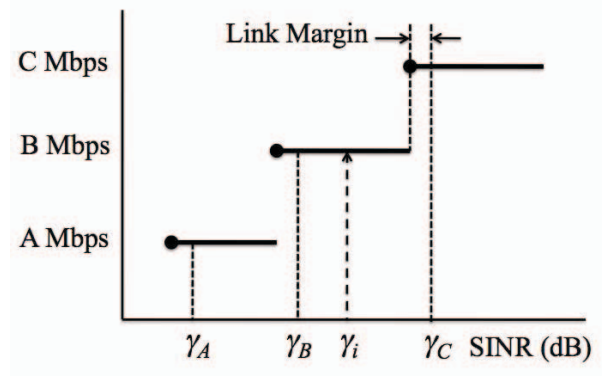

Fig. 3. Target SINR: Calculate utility and set the target SINR based the staircase utility function

To avoid the problem caused by setting the power value to an optimal point that produce abrupt change in the utility value, in each iteration, power $p_{i}$ is only slightly adjusted by the amount of $\Delta p$ according to the direction that each node achieves higher utility value. Figure. 3 shows how $p_{i}$ is determined based on the current SINR value, $\gamma_{i}$. With current $\gamma_{i}$ between $\gamma_{B}$ and $\gamma_{C}$, each node calculates its utility value assuming the $\gamma_{i}$ equals to $\gamma_{A}$ and $\gamma_{C}$. Then they compare their utility values to the current utility value, and determine the direction of power adjustment.

This utility calculation can be made without exchanging any information with other nodes as the problem is decomposed in a standard way using Lagrangian method. The utility is only dependent on individual node's transmission power, $p_{i}$, of the link $i$. The aggregate interference from all other transmitting nodes, $R_{i}=\sum_{j \neq i} F_{i j} p_{i}+\eta_{i}$ can be calculated from known $p_{i}$, $G_{i i}$, and the amount of thermal noise component. ${ }^{2}$ Algorithm 1 shows the outline of the power control process assuming the receiver node runs the power control algorithm and updates the power information to the transmitter node.

Assuming dynamic channel variations due to fading, the link margin is added to each SINR threshold for the reliable reception of packets. This margin is an environment dependent value, which should be adjusted according to the amount of small scale channel fluctuations. For utility function, it is possible to apply softly varying Sigmoidal function instead of the threshold based staircase function that abruptly switches data rate. However, given the abruptly changing packet error rate at SINR thresholds for small changes in SINR, the impact on total utility values from Sigmoidal utility function is marginal. Also it over complicates the algorithm.

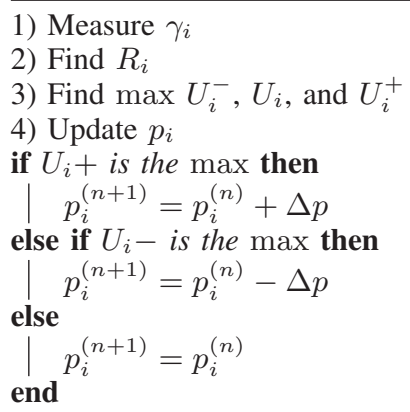

5) receiver node $r_{i}$ feedback $p_{i}^{(n+1)}$ to transmitter node $s_{i}$ 6) Update $\lambda^{(n+1)}$

$$
\begin{aligned}
& \lambda_{i}^{(n+1)} \\
& k=k+1
\end{aligned}=\left[\lambda_{i}^{(n)}+\frac{\alpha}{k}\left(p_{i}^{(n+1)}-\bar{p}_{i}\right)\right]^{+}
$$

Algorithm 1: Receiver node feedback: updated power value to the transmitter node

\section{B. Joint power control and scheduling}

We solve the fairness issue discussed in Sect. III-B by jointly scheduling the transmission timing of nodes with power control algorithm simultaneously. However, as mentioned in the previous section, the problem of jointly scheduling and

\footnotetext{
${ }^{2}$ Information on $p_{i}$ is delivered to the receiver node in each packet header, and channel gain $G_{i i}$ can be estimated from RSSI information
} 
power control is a well-known NP-complete problem, which cannot be easily solved in distributed way even with the full exchange of information on each node's status.

For scheduling, JPSA groups node into power control subgroups to reduce mutual interference between nodes so that each subgroup has a feasible solution on their power allocation. The algorithm is designed to based on the following principles.

- The message exchange between nodes should be minimized

- The convergence of power control algorithm should be fast regardless of number of nodes

- The complexity of algorithm should be independent from the size of the rate set

- Each power control subgroup should have a convex rate region

- None of the node should suffer from severe unfairness

We exploit convergence of Lagrangian based power control algorithm in grouping nodes and splitting the channels. Figure 4 show the scheduling algorithm and how nodes are grouped, where we split channel in time domain. The power control process start from the entire node group $C$. When the power control algorithm converges, the nodes in active transmission are grouped to $C_{1}$, and nodes turned off after the power control process initiate the scheduling process. The scheduling process splits the time slot into half by broadcasting a channel split request message. Then they organize the second node subgroup $C_{2}$. The power control algorithm continues to run on subgroup $C_{2}$ and produce another subgroup $C_{3}$ when any node in $C_{2}$ converges to zero transmission power. The scheduling process continues until none of the nodes have zero transmission power, and the channel is equally divided into $k$ channels in time domain.

In the power control process, links under high interference from transmitters of other links are usually turned off as their utility function is hard to grow compared to other links. This is not an optimal solution because the amount of utility is not considered in the process of the decision for splitting nodes into subgroups. However, the scheduling algorithm is simple and fully distributed. The algorithm operates in each node without any coordination among nodes, and it performs reasonably well compared to the optimal scheduling cases requiring a global knowledge on the network. In the next section, through simulations, we show that by allowing time division multiplex and power control simultaneously, we can efficiently increase both the fairness and the network throughput of the Wi-Fi Ah-hoc system.

\section{Simulation Setup}

The channel model used in the simulation is the log-distance path model in (9) with a path-loss exponent value of $\zeta=4$.

$$
L=L_{0}+10 \zeta \log _{10} \frac{d}{d_{0}}
$$

Thermal Gaussian noise on the receiver antenna front-end can be found by the Boltzmann equation, $N=k T B$, where

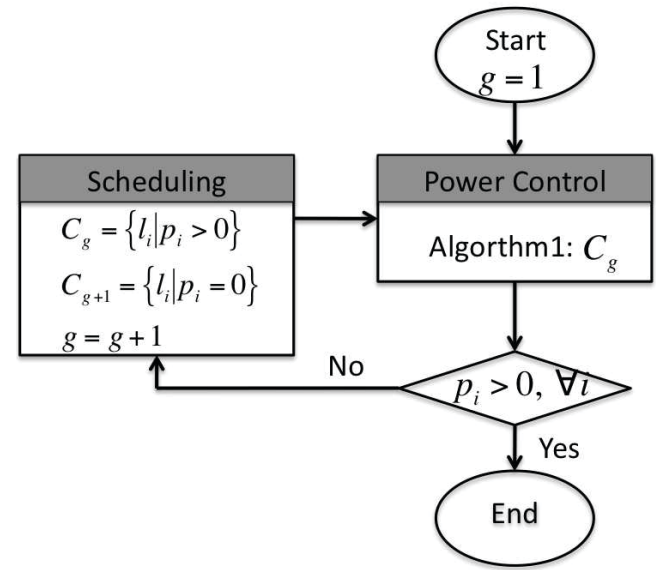

(a) Scheduling algorithm split groups until all the nodes has positive transmission power $\left(p_{i}>0, \forall i\right)$

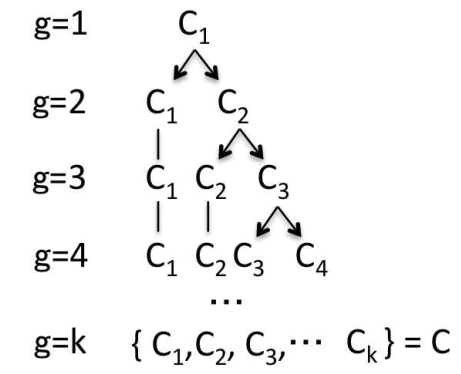

(b) Number of groups increase at each scheduling step

Fig. 4. Joint power control and scheduling algorithm

TABLE I

802.11A RADIO RECEPTION THRESHOLDS: RECEIVER PERFORMANCE REQUIREMENTS $\left(P E R=10^{-3}\right)$

\begin{tabular}{|c|c|c|c|}
\hline Data rate & Modulation & Coding rate & Threshold \\
\hline $6 \mathrm{Mbps}$ & BPSK & $1 / 2$ & $-82 \mathrm{dBm}$ \\
$9 \mathrm{Mbps}$ & BPSK & $3 / 4$ & $-81 \mathrm{dBm}$ \\
$12 \mathrm{Mbps}$ & QPSK & $1 / 2$ & $-79 \mathrm{dBm}$ \\
$18 \mathrm{Mbps}$ & QPSK & $3 / 4$ & $-77 \mathrm{dBm}$ \\
$24 \mathrm{Mbps}$ & 16QAM & $1 / 2$ & $-74 \mathrm{dBm}$ \\
$36 \mathrm{Mbps}$ & 16QAM & $3 / 4$ & $-70 \mathrm{dBm}$ \\
$48 \mathrm{Mbps}$ & 64QAM & $2 / 3$ & $-66 \mathrm{dBm}$ \\
$54 \mathrm{Mbps}$ & 64QAM & $3 / 4$ & $-65 \mathrm{dBm}$ \\
\hline
\end{tabular}

$k$ stands for the Boltzmann's constant equals to $1.38065 \times$ $10^{-8}[\mathrm{~J} / \mathrm{K}], T$ is the effective temperature in kelvin, and $B$ is the bandwidth. In this paper, through the calibration process in using actual Wi-Fi radios we adjust the thresholds for each data rate, which is addressed in Sect.VII in detail.

Table I shows the reception threshold of 802.11a radios for each data rate. Depending on the modulation schemes and convolutional code rate, the sensitivity over noise and interference is determined. The thermal Gaussian noise $N_{i}$ in each receiver incorporates $10 \mathrm{~dB}$ noise figure and $9 \mathrm{~dB}$ loss margin, which adds up to $-87 \mathrm{dBm}$. Based on the channel model in (9), the resulting distance and data rate relationship of ideal 802.11a radios is shown in Fig. 5. 

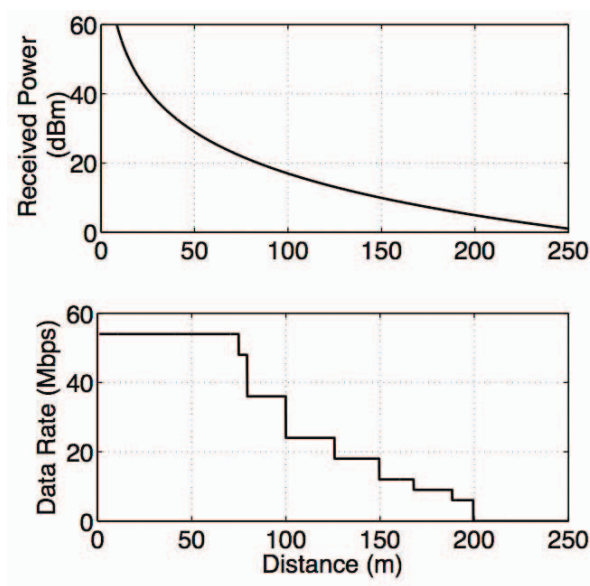

Fig. 5. Distance and data rate: Maximum communication range is set as $200 \mathrm{~m}$ with maximum transmission power set as $\bar{p}_{i}=18 \mathrm{dBm}$

\section{Simulation Results}

In this section, we demonstrate the performance of the proposed JPSA through simulations. Because both the network throughout and the fairness of user are important, we uses a new metric in quantifying the performance of the proposed algorithm. The performance of the algorithm, $P$, is measured by multiplying the fairness of user throughput and the aggregate network throughput. The fairness is the average value of the Jain's fairness index over the link throughputs, $t_{i}$, in a network of total $L$ links.

$$
\operatorname{Performance}(P)=\sum_{i}^{L} t_{i} \times \frac{\left(\sum_{i}^{L} t_{i}\right)^{2}}{L \sum_{i}^{L} t_{i}^{2}}
$$

\section{A. Power control only vs Scheduling only}

Figure 7 shows the simulation result for the example of 5link topology illustrated in Fig. 6. The transmission powers of $l_{1}, l_{2}$, and $l_{4}$ converge to the value that maximizes the sum of data rates of all links. However, $l_{3}$ and $l_{5}$ experience serious unfairness as they are completely turned off. If the deactivated nodes are turned on to alleviate fairness problem, then the system becomes unstable and the power control algorithm diverges to the maximum value resulting in a situation where none of the links get any reasonable throughput.

In this topology, the throughput of the simple power control method is $84 \mathrm{Mbps}$, and the ideal throughput of the contention based CSMA MAC of 802.11 system is $49.2 \mathrm{Mbps}$. Ideal CSMA MAC is identical to the ideal TDMA system as it assumes zero overhead from collisions and back-off processes. In terms of aggregate throughput, TDMA based MAC that only schedules transmission of nodes performs much worse than a power control mechanism with a rate adaptation algorithm. However, the system based on the power control mechanism has serious problem in the fairness as some of links are simply turned off to increase the aggregate network throughput.

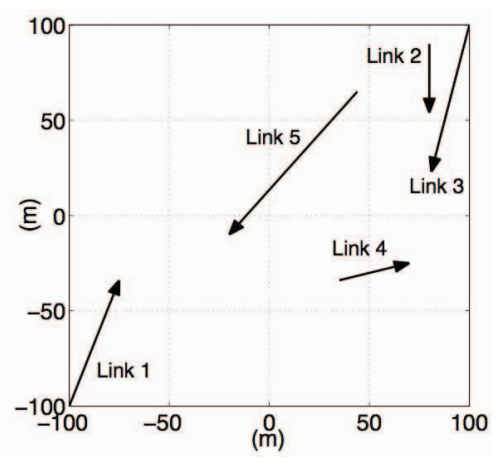

Fig. 6. Example topology: Five links
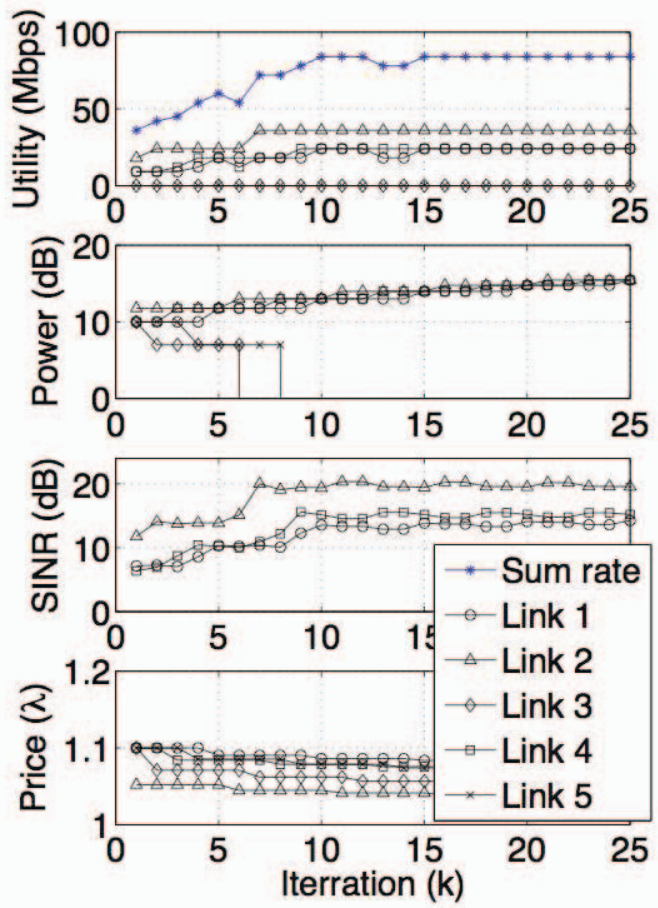

Fig. 7. Power control with a rate adaptation

\section{B. Performance of JPSA: Comparison with fair scheduling algorithm}

The proposed JPSA can solve the fairness problem caused by feasibility issue in the utility based power control algorithm. By grouping links to reduce mutual interference and assigning different slots for each of groups while simultaneously controlling the transmission powers of the links, it is possible to achieve gains in both the aggregate network throughput and the fairness in each user throughout.

In Fig. 8, we compare the performance of JPSA over the TDMA system. TDMA system stands for a system that provides fair time sharing of the channel for all nodes without power control. We generated 5,000 random topologies in $1000 \times 1000 \mathrm{~m}$ space, and the $s_{i}-r_{i}$ distance is limited as $50-150 \mathrm{~m}$. As discussed in the previous section, JPSA shows better performance in aggregate network throughput 
while TDMA shows better fairness. However, in terms of the joint performance $P$, which is a combined performance of both the network throughput and fairness, JPSA performs far better than TDMA especially for the topologies that JPSA converges to small number of subgroups.
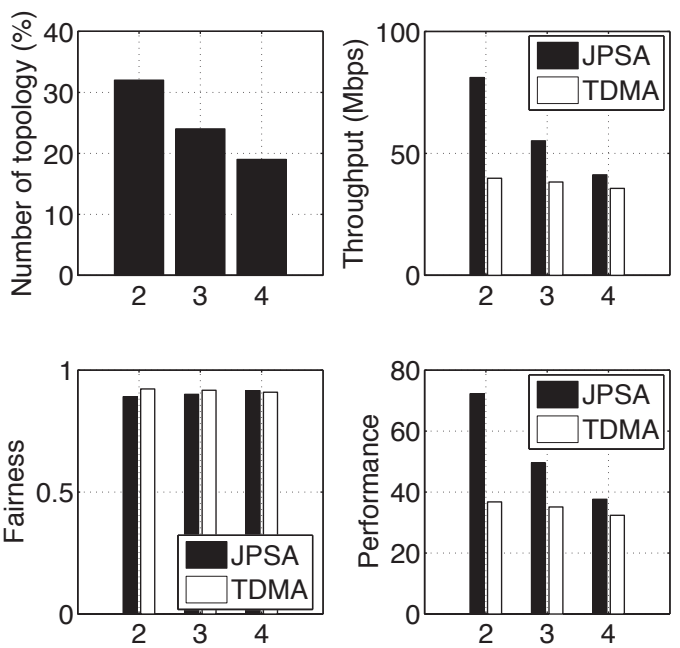

Number of Groups (slots)

Number of Groups (slots)

Fig. 8. Performance comparison with Time division access (TDMA) : 5 Links topology

\section{Performance of JPSA: Comparison with optimal schedul- ing algorithm}

Depending on the topological conditions mutual interference level changes, and the optimal number of subgroup varies, which maximizes the network aggregate throughput. In JPSA, the selection of the number of subgroup and the node assignment for each subgroup is made in a greedy way until all the nodes in the network have positive transmission power. As JPSA is a suboptimal algorithm, there exist a performance gap to the optimal scheduling method that ideally selects the number of subgroup and rightly assigns nodes to each subgroup for maximum throughput. In this paper, the optimal performance is found by an exhaustive search algorithm that runs power control algorithm for all the different combinations of group assignment cases, which assumes a central coordinator has a global knowledge on the network.

In Fig. 9 the performance of JPSA is compared with the optimal performance. This figure drawn from 1,200 cases that JPSA converges to 3 subgroups, and its performance is sorted according to the descending order of the optimal performance. This figure shows the gap between the optimal scheduling performance and JPSA. We also compare the performance of JPSA to the fair time sharing TDMA performance without power control in Fig. 10, which is sorted according to the descending order of the TDMA MAC performance.

JPSA performs lower than optimal scheduling algorithm. However, considering the complexity of algorithms and the amount of message exchanges required, JPSA performs reasonably well compared to TDMA MAC.

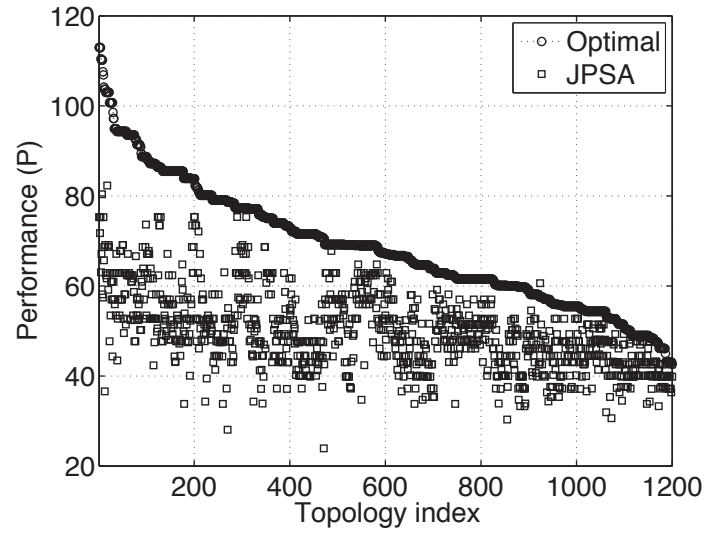

Fig. 9. Performance comparison with an optimal scheduling algorithm: 5 Links topology

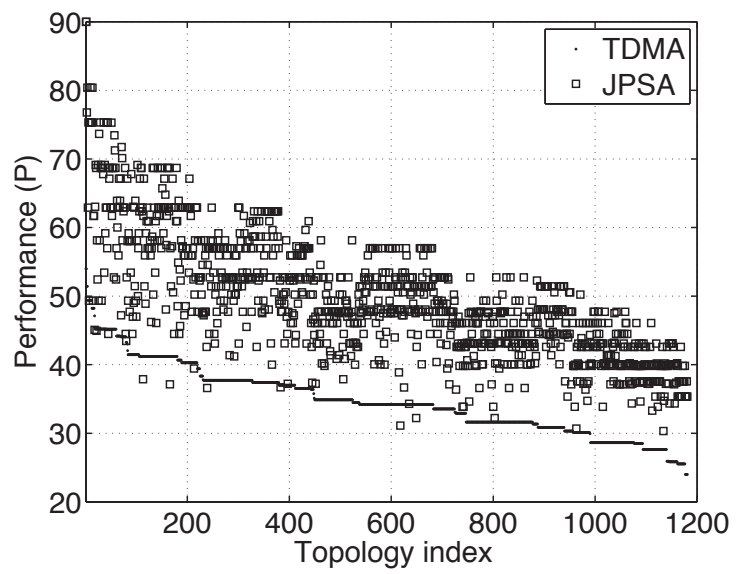

Fig. 10. Performance comparison with a fair channel scheduling algorithm (TDMA): 5 Links topology

\section{EXPERIMENTAL PROOF ON WI-FI SINR MODEL}

JPSA can be applied to any radio systems that are interference limited. In this section, before applying power control to packet based Wi-Fi systems, we firstly verify wether commodity 802.11 radios follow standard SINR models like other interference limited systems such as CDMA or OFDMA systems. When two packets collide, according to standard SINR models, radios can decode the strongest frame if its SINR exceeds a given threshold. The threshold is usually derived from the modulation and coding scheme assuming AWGN interference. To fully exploit power control techniques in Wi-Fi systems, the SINR model for synchronous packet collisions of real network cards should be verified by experiments. Then measured SINR thresholds is also used for the calibration of simulations.

We verify the SINR model of Wi-Fi radios by artificially generating power capture effects from synchronous packet collisions and measuring the capture thresholds. Power capture indicates cases that more than two packets collide in the air, and a packet with stronger energy can be successfully decoded 
in the receiver [22]. There are several approaches to extend this effects to increase network throughputs. However, none of them analyzed power capture in the context of SINR modeling for the power control mechanism.

\section{A. Experiment setup}

The primary goal of this experiment is to verify the SINR model of 802.11 radios. Measurement uses the ORBIT experimental setup [23]. ORBIT nodes are equipped with two Z-Com miniPCI cards based on the Atheros 5212 chipset(supporting $802.11 \mathrm{a} / \mathrm{b} / \mathrm{g}$ ). To establish the accuracy of these readings and confirm interpretation of the RSSI values, we calibrated experiment radios using a Vector Signal Analyzer (VSA). After the calibration, we proceed to the experiments for the packet capture thresholds. A large number of packet collision are artificially generated to measure the threshold for the capture effect. While we are not able to deterministically generate collisions, we eased the interpretation of the results by increasing the probability of collisions by choosing the smallest possible minimum contention window size $(\mathrm{CWmin}=7)$ on the Atheros chip. ${ }^{3}$ We also saturated the channel using the "-flood"option in the Hping application.

\section{B. Power capture threshold from a single interference}

Using a 3-node topology from the ORBIT grid, we experiment with power capture in 802.11a system. One of the transmitting node $s_{i}$ has a fixed transmission power while the other interfering node $s_{j}$ linearly increases its transmission power. Both transmitters use $6 \mathrm{Mbps}$ data rate.

When two transmitters have similar transmission power, colliding packets will be lost at the receiver node $r_{i}$, resulting in a packet reception rate (PRR) close to one. As the SINR approaches the capture threshold, however, stronger packets are captured by $r_{i}$ and PRR approaches 100\%. Fig. 11(a) shows the measured capture threshold over three different transmitter and interferer combinations.

We can derive the capture threshold from this PRR curve. The threshold is $5 \mathrm{~dB}$ for $70 \%$ capture and $6 \mathrm{~dB}$ for $90 \%$ capture. We have applied the scale of RSSI-dB relationship from the node calibration process in calculating the RSSI difference in $[\mathrm{dBm}]$. Because only relative RSSI is used, the RSSI offsets do not affect this measurement result. Thus, these results can be interpreted with the same $0.3 \mathrm{~dB}$ confidence intervals established in the prior calibration experiments.

\section{Power capture threshold from a multiple interference}

Fig. 11(b) shows the capture threshold for more than two colliding packets. The RSSI difference is calculated using Eq.1 with the consideration for the standard thermal Gaussian noises in 802.11a radio devices. The sum of interfering powers from the interfering nodes are calculated by individually measured RSSI from each interferer at the receiver. Then, the aggregated interfering power is subtracted from the transmitter's signal power. This shows that the interferences power from interfering nodes linearly adds unlike the case of low

\footnotetext{
${ }^{3}$ This value appears to be limited by hardware.
}

power devices in [8]. This proves that the existing SINR model of Eq.1 is valid over mid-high power Wi-Fi devices with a properly specified capture threshold values, which can be measured by the capture experiment.

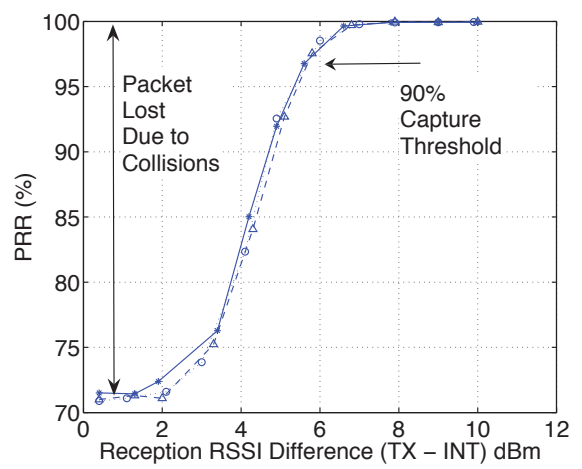

(a) Power Capture: One interferer

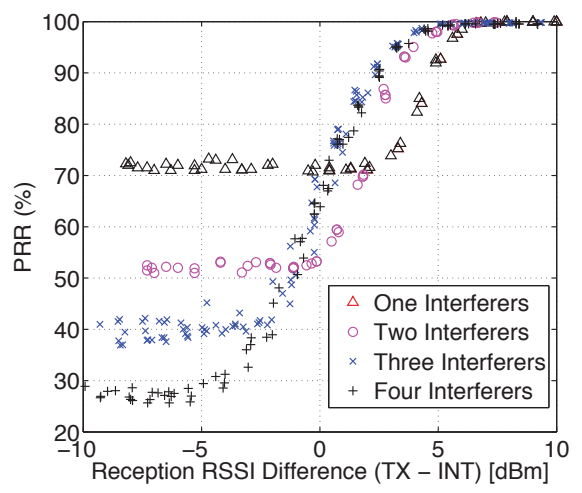

(b) Power Capture: More than one interferers

Fig. 11. Power Capture: The percentage of captured packet over the reception power difference at the receiver node

The next step is to find the capture threshold over various data rates. In Fig. 12, we can verify higher order modulation has higher SINR threshold, and the thresholds approximately match with ideal values found by the modulation and coding schemes against AWGN environments.

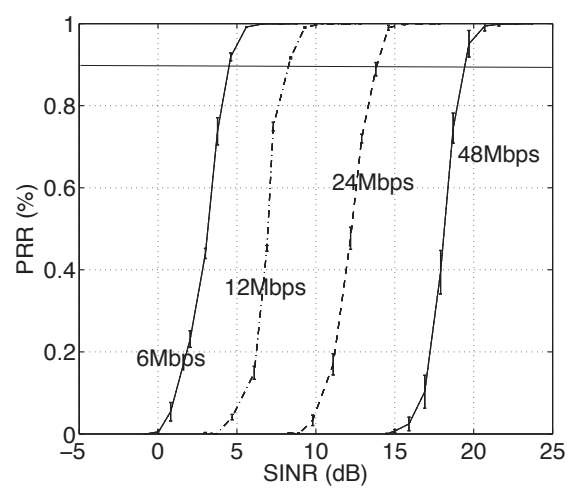

Fig. 12. SINR Threshold for various data rates 


\section{CONCLUSIONS AND Discussions}

In this paper, we introduced a joint scheduling and power control algorithm for Wi-Fi systems. Considering the need for peer to peer communications among mobile devices and the popularity of 802.11 protocol based $\mathrm{Wi}-\mathrm{Fi}$ radios, it is a meaningful approach to increase the network throughput of Wi-Fi systems by a relatively simple and distributed power control algorithm where individual nodes socially adjust their transmission power.

To solve the fairness problems that arise in the power control mechanism, we introduced a simple greedy scheduling algorithm which is non-optimal, but performs reasonably well even without the need for the exchange of information on the mutual status between nodes. Moreover, we showed the power control schedules can be applied to Wi-Fi devices by experimentally verifying the SINR model from synchronous packet collisions using stock 802.11a network cards. We made a precise modeling of packet reception under co-channel interference for $\mathrm{Wi}-\mathrm{Fi}$ radios by measuring frame error rate under collisions with interferers of different power levels.

For an actual implementation of the proposed JPSA algorithm in Wi-Fi systems, back-off process of 802.11 CSMA MAC should be completely controllable by software. Although there has been a number of approaches for this by tweaking open-source drivers, to our best knowledge, there is no reliable solution that completely controls the MAC of 802.11 radios with the current version of open source drivers. We expect running the proposed JPSA algorithm in real world Wi-Fi networks with future updated drivers.

Our contribution can be summarized as follows.

- The potential capacity expansion of $\mathrm{Wi}-\mathrm{Fi}$ radios is highlighted, which can be enabled by NUM based power control mechanism

- A joint power control and scheduling algorithm is provided solving the fairness problems. It has a linear complexity with number of nodes and is independent from the size of rate set

- Simulation results are realistic as SINR thresholds are calibrated by real Wi-Fi network cards

- The SINR model for $\mathrm{Wi}-\mathrm{Fi}$ radios is experimentally verified, which is required in applying power control mechanism to Wi-Fi systems

\section{REFERENCES}

[1] Mingbo Xiao, Ness B. Shroff, and Edwin K. P. Chong. A utility-based power-control scheme in wireless cellular systems. IEEE Transactions on Networking, 11(2):210-221, 2003.

[2] Cem U. Saraydar, Narayan B. Mandayam, and David J. Goodman. Efficient power control via pricing in wireless data networks. IEEE Transactions on Communication, 2000.

[3] Hongbin Ji and Ching-Yao Huang. Non-cooperative uplink power control in cellular radio systems. Proc. of Wireless Networks, 4(3):233240,1998

[4] P. Hande, S. Rangan, and M. Chiang. Distributed uplink power control for optimal sir assignment in cellular data networks. pages 1-13, April 2006.

[5] Xiaojun Lin and N.B. Shroff. Joint rate control and scheduling in multihop wireless networks. In Proc. of IEEE Decision and Control, volume 2, pages 1484-1489 Vol.2, Dec. 2004.
[6] Bozidar Radunovic and Jean-Yves Le Boudec. Joint Scheduling, Power Control and Routing in Symmetric, One-dimensional, Multihop Wireless Networks. In WiOpt'03: Modeling and Optimization in Mobile,Ad Hoc and Wireless Networks, 2003.

[7] Min Cao, V. Raghunathan, S. Hanly, V. Sharma, and P.R. Kumar. Power control and transmission scheduling for network utility maximization in wireless networks. In Proc. of Decision and Control, Dec. 2007.

[8] Dongjin Son, Bhaskar Krishnamachari, and John Heidemann. Experimental study of concurrent transmission in wireless sensor networks. In SenSys '06, pages 237-250, 2006.

[9] G.J. Foschini and Z. Miljanic. A simple distributed autonomous power control algorithm and its convergence. IEEE Transactions on Vehicular Technology, 42(4):641-646, Nov 1993.

[10] Sang wook Han, Hoon Kim, and Youngnam Han. Distributed utilitymaximization using a resource pricing power control in uplink ds-cdma. Communications Letters, IEEE, 12(4):286-288, April 2008.

[11] Kishore Ramachandran, Ravi Kokku, Honghai Zhang, and Marco Gruteser. Symphony: synchronous two-phase rate and power control in 802.11 wlans. In Proc. of Mobile systems, applications, and services (MobiSys), pages 132-145. ACM, 2008.

[12] J. P. Monks, V. Bharghavan, and W. M. W. Hwu. A power controlled multiple access protocol for wireless packet networks. In Proc. of IEEE INFOCOM, volume 1, pages 219-228 vol.1, 2001.

[13] Alaa Muqattash and Marwan Krunz. A single-channel solution for transmission power control in wireless ad hoc networks. In Proc. of Mobile ad hoc networking and computing (MobiHoc), pages 210-221, New York, NY, USA, 2004. ACM.

[14] A. Sheth and R. Han. Shush: reactive transmit power control for wireless mac protocols. In Proc. of Wireless Internet Conference (WICON, pages 18-25, July 2005

[15] Vasudev Shah and Srikanth Krishnamurthy. Handling asymmetry in power heterogeneous ad hoc networks: A cross layer approach. Proc. of Distributed Computing Systems, 0:749-759, 2005.

[16] Aditya Akella, Glenn Judd, Srinivasan Seshan, and Peter Steenkiste. Self-management in chaotic wireless deployments. In Proc. of Mobile computing and networking (MobiCom), pages 185-199. ACM Press, 2005.

[17] Tae-Suk Kim, Hyuk Lim, and Jennifer C. Hou. Improving spatial reuse through tuning transmit power, carrier sense threshold, and data rate in multihop wireless networks. In Procc of Mobile computing and networking (MobiCom), pages 366-377, New York, NY, USA, 2006. ACM.

[18] Daji Qiao, Sunghyun Choi, Amit Jain, and Kang G. Shin. Miser: an optimal low-energy transmission strategy for ieee $802.11 \mathrm{a} / \mathrm{h}$. In Proc. Mobile computing and networking (MobiCom), pages 161-175. ACM, 2003.

[19] V.P. Mhatre, K. Papagiannaki, and F. Baccelli. Interference mitigation through power control in high density 802.11 wlans. In Proc. of IEEE INFOCOM, pages 535-543, May 2007.

[20] S Narayanaswamy, V Kawadia, R.S Sreenivas, and P.R Kumar. Power control in ad-hoc networks: Theory, architecture, algorithm and implementation of the compow protocol. In Proc. of European Wireless, 2002.

[21] M. Johansson and L. Xiao. Cross-layer optimization of wireless networks using nonlinear column generation. Wireless Communications, IEEE Transactions on, 5(2):435-445, Feb. 2006.

[22] Jeongkeun Lee, Wonho Kim, Sung-Ju Lee, Daehyung Jo, Jiho Ryu, Taekyoung Kwon, and Yanghee Choi. An experimental study on the capture effect in 802.11a networks. In Proc. of Wireless network testbeds, experimental evaluation and characterization (WinTECH), pages 19-26, New York, NY, USA, 2007. ACM.

[23] D. Raychaudhuri, I. Seskar, M. Ott, S. Ganu, K. Ramachandran, H. Kremo, R. Siracusa, H. Liu, and M. Singh. Overview of the ORBIT radio grid testbed for evaluation of next-generation wireless network protocols. In $W C N C, 2005$. 